\title{
Properties of Some Partial Dynamic Equations on Time Scales
}

\author{
Deepak B. Pachpatte \\ Department of Mathematics, Dr. B. A. M. University, Aurangabad, Maharashtra 431004, India
}

Correspondence should be addressed to Deepak B. Pachpatte; pachpatte@gmail.com

Received 23 February 2013; Revised 6 June 2013; Accepted 16 June 2013

Academic Editor: Anjan Biswas

Copyright (C) 2013 Deepak B. Pachpatte. This is an open access article distributed under the Creative Commons Attribution License, which permits unrestricted use, distribution, and reproduction in any medium, provided the original work is properly cited.

The main objective of the paper is to study the properties of the solution of a certain partial dynamic equation on time scales. The tools employed are based on the application of the Banach fixed-point theorem and a certain integral inequality with explicit estimates on time scales.

\section{Introduction}

Recently, there has been a lot of interest in shown studying various properties of dynamic equations on time scales by various authors [1-11]. In this paper, we study some partial dynamic equations on time scales. Let $\mathbb{R}^{n}$ denote the $n$ dimensional Euclidean space with appropriate norm $|\cdot|$. In this, let $\mathbb{R}$ denote the set of real numbers, $\mathbb{Z}$ the set of integers, and $\mathbb{T}$ the arbitrary time scales. Let $\mathbb{T}_{1}$ and $\mathbb{T}_{2}$ be two time scales, and let $\Omega=\mathbb{T}_{1} \times \mathbb{T}_{2}$. Let $C_{\text {rd }}$ denote the set of rdcontinuous function. We assume basic understanding of time scales and notations. More information about time scales calculus can be found in [12-14]. The partial delta derivative of $z(x, y)$ for $(x, y) \in \Omega$ with respect to $x, y$, and $x y$ is denoted by $z^{\Delta_{1}}(x, y), z^{\Delta_{2}}(x, y)$, and $z^{\Delta_{1} \Delta_{2}}(x, y)=z^{\Delta_{2} \Delta_{1}}(x, y)$.

Many physical systems can be modeled using dynamical systems on time scales. As response to the needs of diverse applications, many authors have studied qualitative properties of various equations on time scales [4-9, 11]. Motivated by the results in this paper, I consider the partial dynamic equation of the form

$$
u^{\Delta_{2} \Delta_{1}}(x, y)=f\left(x, y, u(x, y), u^{\Delta_{2} \Delta_{1}}(x, y),(H u)(x, y)\right)
$$

with the initial boundary conditions

$$
u\left(x, x_{0}\right)=\alpha(x), \quad u\left(y_{0}, y\right)=\beta(y), \quad u\left(x_{0}, y_{0}\right)=0,
$$

for $(x, y) \in \Omega$, where

$$
\begin{aligned}
& (H u)(x, y) \\
& =\int_{x_{0}}^{x} \int_{y_{0}}^{y} g\left(x, y, m, n, u(m, n), u^{\Delta_{2} \Delta_{1}}(x, y)\right) \Delta n \Delta m,
\end{aligned}
$$

$f \in C_{\mathrm{rd}}\left(\Omega \times \mathbb{R}^{n} \times \mathbb{R}^{n} \times \mathbb{R}^{n}, \mathbb{R}^{n}\right), g \in C_{\mathrm{rd}}\left(\Omega \times \Omega \times \mathbb{R}^{n} \times\right.$ $\left.\mathbb{R}^{n}, \mathbb{R}^{n}\right), \alpha, \beta \in C_{\mathrm{rd}}\left(\mathbb{T}, \mathbb{R}^{n}\right)$, and $\left(H_{0}\right)(x, y)=\int_{x_{0}}^{x} \int_{y_{0}}^{y} g(x$, $y, m, n, 0,0) \Delta n \Delta m$.

\section{Preliminaries and Basic Inequality}

We now give some basic definitions and notations about time scales. Define the jump operators $\sigma, \rho: \mathbb{T} \rightarrow \mathbb{T}$ by

$$
\sigma(t)=\inf \{s \in \mathbb{T}: s>t\}, \quad \rho(t)=\sup \{s \in \mathbb{T}: s<t\}
$$

If $\sigma(t)=t$ and $\sigma(t)<t$, then the point $t \in \mathbb{T}$ is left dense and left scattered. If $\rho(t)=t$ and $\rho(t)<t$, then the point $t \in \mathbb{T}$ is right-dense and right-scattered. If $\mathbb{T}$ has a right scattered minimum $m$, define $\mathbb{T}^{k}:=\mathbb{T}-m$; otherwise, $\mathbb{T}^{k}:=\mathbb{T}$. If $\mathbb{T}$ has a left-scattered maximum $M$, define $\mathbb{T}^{k}:=\mathbb{T}-M$; otherwise, $\mathbb{T}^{k}:=\mathbb{T}$. The graininess function $\mu: \mathbb{T} \rightarrow R_{+}=[0, \infty)$ is defined by $\mu(t)=\sigma(t)-t$. We say that $p: \mathbb{T} \rightarrow \mathbb{R}$ is regressive provided $1+\mu(t) p(t) \neq 0$ for all $t \in \mathbb{T}$. For $f: \mathbb{T} \rightarrow R$ and $t \in \mathbb{T}^{k}$, the delta derivative of $f$ at $t$ denoted by $f^{\Delta}(t)$ is the 
number (provided it exists) with the property that given any $\epsilon>0$, there is a neighborhood $U$ of $t$ such that

$$
\left|f(\sigma(t), \tau)-f(s, \tau)-f^{\Delta}(t, \tau)(\sigma(t)-s)\right| \leq \epsilon|\sigma(t)-s|,
$$

for all $s \in U$. For $\mathbb{T}=R, f^{\Delta}(t)=f^{\prime}(t)$ the usual derivative; for $\mathbb{T}=Z$, the delta derivative is the forward difference operator, $f^{\Delta}(t)=f(t+1)-f(t)$. A function $f: \mathbb{T} \rightarrow R$ is rightdense continuous or rd-continuous provided it is continuous at right-dense points in $\mathbb{T}$ and its left-sided limits exist (finite) and at left-dense points in $\mathbb{T}$ and its left-sided limit exists (finite) at left-dense points in $\mathbb{T}$. If $\mathbb{T}=R$, then $f$ is rdcontinuous if and only if $f$ is continuous. It is known [12, Theorem 1.74] that $f$ is right-dense continuous, there is a function $F$ such that $f^{\Delta}(t)=f(t)$ and

$$
\int_{a}^{b} f(t) \Delta t=F(b)-F(a),
$$

where $a, b \in \mathbb{T}$. Note that when $\mathbb{T}=R, \sigma(t)=t, \mu(t)=0, f^{\Delta}=$ $f^{\prime}$, and $\int_{a}^{b} f(t) \Delta t=\int_{a}^{b} f(t) \mathrm{d} t$, while then $\mathbb{T}=Z, \sigma(t)=t+1$, $\mu(t)=1, f^{\Delta}=\Delta f$, and $\int_{a}^{b} f(t) \Delta t=\sum_{t=a}^{p-1} f(t)$. We denote by $\mathfrak{R}$ the set of all regressive and rd-continuous functions and $\mathfrak{R}^{+}=\{p \in \mathbb{R}: 1+\mu(t) p(t)>0$ for all $t \in \mathbb{T}\}$. For $p \in \mathfrak{R}$, we define (see [12, Theorem 2.35]) the exponential function $e_{p}\left(\cdot, t_{0}\right)$ on time scale $\mathbb{T}$ as the unique solution to the scalar initial value problem

$$
x^{\Delta}=p(t) x, \quad x\left(t_{0}\right)=1 .
$$

If $p \in \mathfrak{R}^{+}$, then $e_{p}\left(t, t_{0}\right)>0$ for all $t \in \mathbb{T}$ (see [12, Theorem 2.41]). As usual, the set of rd-continuous functions is denoted by $C_{\text {rd }}$.

Denote by $|z(x, y)|_{W}=|z(x, y)|+\left|z^{\Delta_{1} \Delta_{2}}(x, y)\right|$. For $(t, s) \in \Omega$; the notation $a(t, s)=O(b(t, s))$; then there exists a constant $q>0$ such that $|a(t, s) / b(t, s)| \leq q$ right hand neighbourhood. Let $G$ be the space with function $\left(\phi(x, y), \phi^{\Delta_{1} \Delta_{2}}(x, y)\right) \in W$ which are rd-continuous for $(x, y) \in \Omega$ and satisfy the condition

$$
|\Phi(x, y)|_{W} \leq O\left(e_{\lambda}(x, y)\right)
$$

for $(x, y) \in \Omega$, where $\lambda>0$ is a constant. In space $G$, define the norm

$$
|\Phi|_{G}=\operatorname{Sup}_{(x, y) \in \Omega}\left[|\Phi(x, y)|_{W} e_{\Theta \lambda}(x, y)\right]
$$

The norm defined in (9) is clearly a Banach space. Then, (8) implies that there is a constant $N \geq 0$ such that

$$
|\Phi(x, y)|_{W} \leq N e_{\lambda}(x, y) .
$$

Using (10), we observe that

$$
|\Phi(x, y)|_{W} \leq N
$$

The solution of (1) and (2) means a function $u(x, y) \epsilon$ $C_{\mathrm{rd}}\left(\Omega, \mathbb{R}^{n}\right)$ satisfying (1) and (2). It is easy to observe that solution $u(x, y)$ of initial boundary value problem (1) and (2) satisfies following dynamic integral equations on time scales:

$$
\begin{aligned}
u(x, y)= & \alpha(x)+\beta(y) \\
& +\int_{x_{0}}^{x} \int_{y_{0}}^{y} f(s, t, u(s, t),(H u)(s, t)) \Delta t \Delta s,
\end{aligned}
$$

for $(x, y) \in \Omega$.

I need the following lemma proved in [15].

Lemma 1. Let $u, a, f \in C_{\mathrm{rd}}\left(\Omega, \mathbb{R}_{+}\right)$with $a$ and $f$ nondecreasing in each of the variables and $g\left(t, t_{1}, s, s_{2}\right) \in C_{\mathrm{rd}}\left(\Omega \times \Omega, \mathbb{R}_{+}\right)$, $a_{1}<s_{1}<t_{1}, a_{2}<s_{2}<t_{2}$. If

$$
\begin{aligned}
& u\left(t_{1}, t_{2}\right) \leq a\left(t_{1}, t_{2}\right) \\
&+f\left(t_{1}, t_{2}\right) \int_{a_{1}}^{t_{1}} \int_{a_{2}}^{t_{2}} g\left(t, t_{1}, s, s_{2}\right) \\
& \quad \times u\left(s_{1}, s_{2}\right) \Delta_{1} s_{1} \Delta_{2} s_{2},
\end{aligned}
$$

for $\left(t_{1}, t_{2}\right) \in \Omega$, then

$$
u\left(t_{1}, t_{2}\right) \leq a\left(t_{1}, t_{2}\right) e_{\int_{a_{2}}^{t_{2}} f\left(t, t_{2}\right) g\left(t, t_{2}, t_{1}, s_{2}\right) \Delta_{2} s_{2}}\left(t_{1}, a_{1}\right),
$$

for $\left(t_{1}, t_{2}\right) \in \Omega$.

Now I prove the following integral inequality which is used in our results.

Lemma 2. Let $w(x, y), a(x, y) \in C_{\mathrm{rd}}\left(\Omega, \mathbb{R}_{+}\right), p(x, y, s, t)$, $b(x, y, s, t), b^{\Delta_{1}}(x, y, s, t), b^{\Delta_{2}}(x, y, s, t), b^{\Delta_{1} \Delta_{2}}(x, y, s, t) \quad \epsilon$ $C_{\mathrm{rd}}\left(\Omega \times \Omega, \mathbb{R}_{+}\right)$, and let $c \geq 0$ be a constant. If

$$
\begin{aligned}
w(x, y) \leq c+\int_{x_{0}}^{x} \int_{y_{0}}^{y}\{a & (s, t) w(s, t) \\
& +b(x, y, s, t) w(s, t) \\
& +\int_{x_{0}}^{s} \int_{y_{0}}^{t} p(s, t, m, n) \\
& \times w(m, n) \Delta n \Delta m\} \Delta t \Delta s
\end{aligned}
$$

for $(x, y) \in \Omega$, then

$$
\begin{gathered}
w(x, y) \leq c e_{\mathrm{Q}(x, y)}\left(x, x_{0}\right), \\
Q(x, y)=\int_{y_{0}}^{y}[a(s, t)+A(s, t)] \Delta t,
\end{gathered}
$$


for $(x, y) \in \Omega$, where

$$
\begin{aligned}
A(x, y)= & b(x, y, x, y)+\int_{x_{0}}^{x} b^{\Delta_{1}}(x, y, m, y) \Delta m \\
& +\int_{y_{0}}^{y} b^{\Delta_{1}}(x, y, x, n) \Delta n \\
& +\int_{x_{0}}^{x} \int_{y_{0}}^{y} b^{\Delta_{1} \Delta_{2}}(x, y, m, n) \Delta n \Delta m \\
& +\int_{x_{0}}^{x} \int_{y_{0}}^{y} p(x, y, m, n) \Delta n \Delta m .
\end{aligned}
$$

Proof. Define a function $z(x, y)$ by the right hand side of (15), then $z\left(x, x_{0}\right)=z\left(y_{0}, y\right)=c, w(x, y) \leq z(x, y)$, and $z(x, y)$ is nondecreasing in $x$ and $y$ :

$$
\begin{aligned}
& z^{\Delta_{1} \Delta_{2}}(x, y) \\
& =a(x, y) w(x, y)+b(x, y, x, y) w(x, y) \\
& +\int_{x_{0}}^{x} b^{\Delta_{1}}(x, y, m, y) w(m, y) \Delta m \\
& +\int_{y_{0}}^{y} b^{\Delta_{2}}(x, y, x, n) w(x, n) \Delta n \\
& +\int_{x_{0}}^{x} \int_{y_{0}}^{y} b^{\Delta_{2} \Delta_{1}}(x, y, m, n) w(m, n) \Delta n \Delta m \\
& +\int_{x_{0}}^{x} \int_{y_{0}}^{y} p(x, y, m, n) w(m, n) \Delta n \Delta m \\
& \leq a(x, y) z(x, y)+b(x, y, x, y) z(x, y) \\
& +\int_{x_{0}}^{x} b^{\Delta_{1}}(x, y, m, y) z(m, y) \Delta m \\
& +\int_{y_{0}}^{y} b^{\Delta_{2}}(x, y, x, n) z(x, n) \Delta n \\
& +\int_{x_{0}}^{x} \int_{y_{0}}^{y} b^{\Delta_{2} \Delta_{1}}(x, y, m, n) z(m, n) \Delta n \Delta m \\
& +\int_{x_{0}}^{x} \int_{y_{0}}^{y} p(x, y, m, n) z(m, n) \Delta n \Delta m \\
& \leq[a(x, y)+b(x, y, x, y) \\
& +\int_{x_{0}}^{x} b^{\Delta_{1}}(x, y, m, y) \Delta m \\
& +\int_{y_{0}}^{y} b^{\Delta_{2}}(x, y, x, n) \Delta n \\
& +\int_{x_{0}}^{x} \int_{y_{0}}^{y} b^{\Delta_{2} \Delta_{1}}(x, y, m, n) \Delta n \Delta m
\end{aligned}
$$

$$
\begin{aligned}
& \left.\quad+\int_{x_{0}}^{x} \int_{y_{0}}^{y} p(x, y, m, n) \Delta n \Delta m\right] z(x, y) \\
& =[a(x, y)+A(x, y)] z(x, y),
\end{aligned}
$$

where $A(x, y)$ is given by (18). Now, by application of Lemma 1, we get

$$
z(x, y) \leq c \int_{y_{0}}^{y}[a(s, t)+A(s, t)] \Delta_{2} t\left(x, x_{0}\right) .
$$

Using (20) in $w(x, y) \leq z(x, y)$, we get the required inequality (16).

\section{Existence and Uniqueness}

Now our main results are as follows.

Theorem 3. Suppose that

(i) the function $f, g$ in (1) satisfies the conditions

$$
\begin{aligned}
& |f(x, y, u, v, w)-f(x, y, \bar{u}, \bar{v}, \bar{w})| \\
& \quad \leq k(x, y)[|u-\bar{u}|+|v-\bar{v}|+|w-\bar{w}|], \\
& |g(x, y, m, n, u, v)-g(x, y, m, n, \bar{u}, \bar{v})| \\
& \quad \leq h(x, y, m, n)[|u-\bar{u}|+|v-\bar{v}|],
\end{aligned}
$$

where $k(x, y) \in C_{\mathrm{rd}}\left(\Omega, \mathbb{R}_{+}\right), h(x, y, m, n) \in C_{\mathrm{rd}}\left(\Omega \times \Omega, \mathbb{R}_{+}\right)$.

(ii) for $\lambda$ as in (10),

(a) there exists a nonnegative constant $\gamma$ such that $\gamma<1$

$$
L(x, y)+\int_{x_{0}}^{x} \int_{y_{0}}^{y} L(s, t) \Delta t \Delta s \leq \gamma e_{\lambda}(x, y),
$$

where

$$
\begin{aligned}
& L(x, y)=k(x, y) e_{\lambda}(x, y) \\
& +\int_{x_{0}}^{x} \int_{y_{0}}^{y} h(x, y, m, n) \\
& \quad \times e_{\lambda}(m, n) \Delta n \Delta m,
\end{aligned}
$$

(b) there exists a nonnegative constant $\eta$ such that

$$
\begin{aligned}
& |\alpha(x)|+|\beta(y)| \\
& +\left|f\left(x, y, x_{0}, y_{0},(H 0)(x, y)\right)\right| \\
& +\int_{x_{0}}^{x} \int_{y_{0}}^{y}\left|f\left(s, t, x_{0}, y_{0},(H 0)(s, t)\right)\right| \Delta t \Delta s \\
& \quad \leq \eta e_{\lambda}(x, y)
\end{aligned}
$$

then, the initial boundary value problem (1) and (2) has a unique solution on $\Omega$. 
Proof. Let $u(x, y) \in G$, and define the operator $S$ by

$$
\begin{array}{r}
(\mathrm{Su})(x, y)=\alpha(x)+\beta(y) \\
+\int_{x_{0}}^{x} \int_{y_{0}}^{y} f\left(s, t, u(s, t), u^{\Delta_{2} \Delta_{1}}(s, t),\right. \\
(H u)(s, t)) \Delta t \Delta s .
\end{array}
$$

By delta differentiating (25) with respect to $x$ and $y$, we get

$$
\begin{aligned}
& (S u)^{\Delta_{2} \Delta_{1}}(x, y) \\
& \quad=f\left(x, y, u(x, y), u^{\Delta_{2} \Delta_{1}}(x, y),(H u)(x, y)\right)
\end{aligned}
$$

Now, we show that $S u$ maps $G$ into itself and $S u$ is rdcontinuous on $\Omega$.

From (25) and (26) and hypothesis, we have

$$
\begin{aligned}
& |(S u)(x, y)|+\left|(S u)^{\Delta_{2} \Delta_{1}}(x, y)\right| \\
& \leq|\alpha(x)|+|\beta(y)| \\
& +\int_{x_{0}}^{x} \int_{y_{0}}^{y} \mid f\left(s, t, u(s, t), u^{\Delta_{2} \Delta_{1}}(s, t),(H u)(s, t)\right) \\
& -f\left(s, t, x_{0}, y_{0},(H 0)(s, t)\right) \mid \Delta t \Delta s \\
& +\int_{x_{0}}^{x} \int_{y_{0}}^{y}\left|f\left(s, t, x_{0}, y_{0},(H 0)(s, t)\right)\right| \Delta t \Delta s \\
& +\mid f\left(x, y, u(x, y), u^{\Delta_{2} \Delta_{1}}(x, y),(H u)(x, y)\right) \\
& -f\left(x, y, x_{0}, y_{0},(H 0)(x, y)\right) \\
& +\left|f\left(x, y, x_{0}, y_{0},(H 0)(x, y)\right)\right| \\
& \leq \eta e_{\lambda}(x, y) \\
& +\int_{x_{0}}^{x} \int_{y_{0}}^{y}\left\{k(s, t)|u(s, t)|_{G}\right. \\
& +\int_{x_{0}}^{s} \int_{y_{0}}^{t} h(s, t, m, n)|u(m, n)|_{G} \\
& \times \Delta n \Delta m\} \Delta t \Delta s \\
& +k(x, y)|u(x, y)|_{1} \\
& +\int_{x_{0}}^{x} \int_{y_{0}}^{y} h(x, y, m, n)|u(m, n)|_{G} \\
& \times \Delta n \Delta m \\
& \leq \eta e_{\lambda}(x, y)+|u|_{G}\left\{k(x, y) e_{\lambda}(x, y)\right. \\
& +\int_{x_{0}}^{x} \int_{y_{0}}^{y} h(x, y, m, n) \\
& \times e_{\lambda}(m, n) \Delta n \Delta m
\end{aligned}
$$$$
+\int_{x_{0}}^{x} \int_{y_{0}}^{y}\left\{k(s, t) e_{\lambda}(s, t)\right.
$$$$
+\int_{x_{0}}^{s} \int_{y_{0}}^{t} h(s, t, m, n)
$$$$
\left.\times e_{\lambda}(m, n) \Delta n \Delta m\right\}
$$$$
\times \Delta t \Delta s\}
$$

$$
\begin{aligned}
& \leq \eta e_{\lambda}(x, y)+N\left\{L(x, y)+\int_{x_{0}}^{x} \int_{y_{0}}^{y} L(s, t) \Delta t \Delta s\right\} \\
& \leq[\eta+N \gamma] e_{\lambda}(x, y) .
\end{aligned}
$$

From (27), it follows that $S u \in G$, thus proving that $S$ maps $G$ into itself.

Now, we verify that $S$ is a contraction map. Let $u(x, y), v(x, y) \in G$. From (25) and (26) and using the hypotheses, we have

$$
\begin{aligned}
& |(S u)(x, y)-(S v)(x, y)|+\left|(S u)^{\Delta_{2} \Delta_{1}}(x, y)-(S v)(x, y)\right| \\
& \leq \int_{x_{0}}^{x} \int_{y_{0}}^{y} \mid f\left(s, t, u(s, t), u^{\Delta_{2} \Delta_{1}}(s, t),(H u)(s, t)\right) \\
& -f\left(s, t, v(s, t), v^{\Delta_{2} \Delta_{1}}(s, t),(H v)(s, t)\right) \\
& \times \Delta t \Delta s \\
& +\mid f\left(x, y, u(x, y), u^{\Delta_{2} \Delta_{1}}(x, y),(H u)(x, y)\right) \\
& -f\left(x, y, v(x, y), v^{\Delta_{2} \Delta_{1}}(x, y),(H v)(x, y)\right) \mid \\
& \leq \int_{x_{0}}^{x} \int_{y_{0}}^{y}\left\{k(s, t)|u(s, t)-v(s, t)|_{G}\right. \\
& +\int_{x_{0}}^{s} \int_{y_{0}}^{t} h(s, t, m, n) \\
& \left.\times|u(m, n)-v(m, n)|_{G} \Delta n \Delta m\right\} \\
& \times \Delta t \Delta s \\
& +k(x, y)|u(x, y)-v(x, y)|_{G} \\
& +\int_{x_{0}}^{x} \int_{y_{0}}^{y} h(x, y, m, n)|u(m, n)-v(m, n)|_{G} \\
& \times \Delta n \Delta m \\
& \leq|u-v|_{G}\left\{k(x, y) e_{\lambda}(x, y)\right. \\
& +\int_{x_{0}}^{x} \int_{y_{0}}^{y} h(x, y, m, n) e_{\lambda}(m, n) \\
& \times \Delta n \Delta m
\end{aligned}
$$




$$
\begin{gathered}
+\int_{x_{0}}^{x} \int_{y_{0}}^{y}\left\{k(s, t) e_{\lambda}(s, t)\right. \\
+\int_{x_{0}}^{s} \int_{y_{0}}^{t} h(s, t, m, n) \\
\left.\times e_{\lambda}(m, n) \Delta n \Delta m\right\} \\
\times \Delta t \Delta s\} \\
=|u-v|_{s}\left\{L(x, y)+\int_{x_{0}}^{x} \int_{y_{0}}^{y} L(s, t) \Delta t \Delta s\right\} \\
\leq \gamma|u-v|_{G} e_{\lambda}(x, y) .
\end{gathered}
$$

From (28), we have

$$
|S u-S v|_{G} \leq \gamma|u-v|_{G} .
$$

Since $\gamma<1$, it follows from Banach fixed-point theorem that $S$ has unique fixed point in $G$. The fixed point of $G$ is the solution of (1) and (2). This completes the proof.

Theorem 4. Suppose that function $f, g$ in (1) satisfies the condition

$$
\begin{gathered}
|f(x, y, u, v, w)-f(x, y, \bar{u}, \bar{v}, \bar{w})| \\
\leq d[|u-\bar{u}|+|v-\bar{v}|+|w-\bar{w}|], \\
|g(x, y, m, n, u, v)-g(x, y, m, n, \bar{u}, \bar{v})| \\
\leq p(x, y, m, n)[|u-\bar{u}|+|v-\bar{v}|],
\end{gathered}
$$

where $d$ is a nonnegative constant such that $d<1$ and $p(x, y, m, n), p^{\Delta_{1}}(x, y, m, n), p^{\Delta_{2}}(x, y, m, n), p^{\Delta_{2} \Delta_{1}}(x$, $y, m, n), \in C_{\mathrm{rd}}\left(\Omega \times \Omega, \mathbb{R}^{n}\right)$. Then, initial boundary value problem (1) and (2) has at most one solution on $\Omega$.

Proof. Let $u_{1}(x, y)$ and $u_{2}(x, y)$ be any two solutions of (1) and (2) and $w(x, y)=\left|u_{1}(x, y)-u_{2}(x, y)\right|+\mid u_{1}^{\Delta_{2} \Delta_{1}}(x, y)-$ $u_{2}^{\Delta_{2} \Delta_{1}}(x, y) \mid$; then by hypotheses we have

$$
\begin{aligned}
& w(x, y) \\
& \begin{array}{l}
\leq \int_{x_{0}}^{x} \int_{y_{0}}^{y} \mid f\left(s, t, u_{1}(s, t), u_{1}^{\Delta_{2} \Delta_{1}}(s, t),\left(H u_{1}\right)(s, t)\right) \\
\quad-f\left(s, t, u_{2}(s, t), u_{2}^{\Delta_{2} \Delta_{1}}(s, t),\left(H u_{2}\right)(s, t)\right) \mid \\
\quad \times \Delta t \Delta s
\end{array}
\end{aligned}
$$

$$
\begin{aligned}
& +\mid f\left(x, y, u_{1}(x, y), u_{1}^{\Delta_{2} \Delta_{1}}(x, y),\left(H u_{1}\right)(x, y)\right) \\
& -f\left(x, y, u_{2}(x, y), u_{2}^{\Delta_{2} \Delta_{1}}(x, y),\left(H u_{2}\right)(x, y)\right) \\
& \leq \int_{x_{0}}^{x} \int_{y_{0}}^{y}\left\{d \left[\left|u_{1}(s, t)-u_{2}(s, t)\right|\right.\right. \\
& \left.+\left|u_{1}^{\Delta_{2} \Delta_{1}}(s, t)-u_{2}^{\Delta_{2} \Delta_{1}}(s, t)\right|\right] \\
& +\int_{x_{0}}^{s} \int_{y_{0}}^{t} p(s, t, m, n) \\
& \times\left[\left|u_{1}(m, n)-u_{2}(m, n)\right|\right. \\
& \left.+\left|u_{1}^{\Delta_{2} \Delta_{1}}(m, n)-u_{2}^{\Delta_{2} \Delta_{1}}(m, n)\right|\right] \\
& \times \Delta n \Delta m\} \Delta t \Delta s \\
& +d\left[\left|u_{1}(x, y)-u_{2}(x, y)\right|\right. \\
& \left.+\left|u_{1}^{\Delta_{2} \Delta_{1}}(x, y)-u_{2}^{\Delta_{2} \Delta_{1}}(x, y)\right|\right] \\
& +\int_{x_{0}}^{x} \int_{y_{0}}^{y} p(x, y, m, n) \\
& \times\left[\left|u_{1}(m, n)-u_{2}(m, n)\right|\right. \\
& \left.+\left|u_{1}^{\Delta_{2} \Delta_{1}}(x, y)-u_{2}^{\Delta_{2} \Delta_{1}}(x, y)\right|\right] \Delta n \Delta m .
\end{aligned}
$$

From (31), we have

$$
\begin{aligned}
& w(x, y) \\
& \begin{array}{l}
\leq \frac{1}{1-d} \int_{x_{0}}^{x} \int_{y_{0}}^{y}[d w(s, t)+p(x, y, s, t) w(s, t) \\
\left.\quad+\int_{x_{0}}^{s} \int_{y_{0}}^{t} p(s, t, m, n) w(m, n) \Delta n \Delta m\right] \\
\times \Delta t \Delta s .
\end{array}
\end{aligned}
$$

Now, a suitable application of Lemma 2 to (32) yields

$$
\left|u_{1}(x, y)-u_{2}(x, y)\right|+\left|u_{1}^{\Delta_{2} \Delta_{1}}(x, y)-u_{2}^{\Delta_{2} \Delta_{1}}(x, y)\right| \leq 0
$$

which implies that $u_{1}(x, y)=u_{2}(x, y)$ for $(x, y) \in \Omega$. Therefore, there is at most one solution of (1) and (2) on $\Omega$.

\section{Properties of Solutions}

The following theorem contains estimates of solutions of (1) and (2). 
Theorem 5. Assume that

$$
\begin{gathered}
|f(x, y, u, v, w)| \leq \gamma[|u|+|v|+|w|], \\
|g(x, y, m, n, u, v)| \leq q(x, y, m, n)[|u|+|v|], \\
|\beta(x)|+|\tau(y)| \leq \eta,
\end{gathered}
$$

where $\gamma, \eta$ are nonnegative constants such that $\eta<1$ and $q(x, y, m, n), q^{\Delta_{1}}(x, y, m, n), q^{\Delta_{2}}(x, y, m, n), q^{\Delta_{2} \Delta_{1}}(x, y, m, n)$, $\in C_{\mathrm{rd}}\left(\Omega \times \Omega, \mathbb{R}^{n}\right)$. If $u(x, y)$ for $(x, y) \in \Omega$ is any solution of (1) and (2); then

$$
|u(x, y)|+\left|u^{\Delta_{2} \Delta_{1}}(x, y)\right| \leq \frac{\eta}{1-\gamma} e_{\overline{\mathrm{Q}}(x, y)}\left(x, x_{0}\right),
$$

where

$$
\bar{Q}(x, y)=\int_{y_{0}}^{y}\left[\frac{\gamma}{1-\gamma}+\bar{A}(s, t)\right] \Delta t,
$$

for $(x, y) \in \Omega$,

$$
\begin{aligned}
\bar{A}(x, y)= & \frac{1}{1-\gamma} q(x, y, m, n) \\
& +\int_{x_{0}}^{x} q^{\Delta_{1}}(x, y, m, y) \Delta m \\
& +\int_{y_{0}}^{y} q^{\Delta_{2}}(x, y, x, n) \Delta n \\
& +\int_{x_{0}}^{x} \int_{y_{0}}^{y} q^{\Delta_{2} \Delta_{1}}(x, y, m, n) \Delta n \Delta m .
\end{aligned}
$$

Proof. Since $u(x, y)$ is a solution of (1) and (2) and by hypothesis, we have

$$
\begin{aligned}
& |u(x, y)|+\left|u^{\Delta_{2} \Delta_{1}}(x, y)\right| \\
& \leq \beta(x)+\tau(y) \\
& +\int_{x_{0}}^{x} \int_{y_{0}}^{y}\left|f\left(s, t, u(s, t), u^{\Delta_{2} \Delta_{1}}(s, t),(H u)(s, t)\right)\right| \\
& \quad \times \Delta t \Delta s \\
& +\left|f\left(x, y, u(x, y), u^{\Delta_{2} \Delta_{1}}(x, y),(H u)(x, y)\right)\right| \\
& \leq \eta+\int_{x_{0}}^{x} \int_{y_{0}}^{y}\left\{\gamma\left[|u(s, t)|+\left|u^{\Delta_{2} \Delta_{1}}(s, t)\right|\right]\right. \\
& \quad+\int_{x_{0}}^{s} \int_{y_{0}}^{t} q(s, t, m, n) \\
& \quad \times\left[|u(m, n)|+\left|u^{\Delta_{2} \Delta_{1}}(m, n)\right|\right] \\
& \quad \times \Delta n \Delta m\} \Delta t \Delta s
\end{aligned}
$$

$$
\begin{aligned}
& +\gamma\left[|u(x, y)|+\left|u^{\Delta_{2} \Delta_{1}}(x, y)\right|\right] \\
& +\int_{x_{0}}^{x} \int_{y_{0}}^{y} q(x, y, m, n)\left[|u(m, n)|+\left|u^{\Delta_{2} \Delta_{1}}(m, n)\right|\right] \\
& \quad \times \Delta n \Delta m .
\end{aligned}
$$

From (40), we have

$$
\begin{gathered}
|u(x, y)|+\left|u^{\Delta_{2} \Delta_{1}}(x, y)\right| \\
\leq \frac{\eta}{1-\gamma} \\
+\frac{1}{1-\gamma} \int_{x_{0}}^{x} \int_{y_{0}}^{y}\left\{\gamma\left[|u(s, t)|+\left|u^{\Delta_{2} \Delta_{1}}(s, t)\right|\right]\right. \\
+q(x, y, s, t)[|u(s, t)| \\
+\int_{x_{0}}^{s} \int_{y_{0}}^{t} q(s, t, m, n) \\
\times\left[|u(m, n)|+\left|u^{\Delta_{2} \Delta_{1}}(m, n)\right|\right] \\
\times \Delta n \Delta m\} \Delta t \Delta s .
\end{gathered}
$$

Now, a suitable application of Lemma 2 to (41) yields (37).

Remark 6. If the estimate obtained in (37) is bounded, then solution $u(x, y)$ of $(1)$ and (2) and also $u^{\Delta_{2} \Delta_{1}}(x, y)$ is bounded on $\Omega$.

The following result deals with the continuous dependence of solution of (1) and (2).

Theorem 7. Assume that the functions $f, g$ in (1) and (2) satisfy the conditions (30). Let $u_{1}(x, y)$ and $u_{2}(x, y)$ be the solutions of (1) with the given initial boundary conditions

$$
\begin{gathered}
u_{1}\left(x, x_{0}\right)=\beta_{1}(x), \quad u_{1}\left(y_{0}, y\right)=\tau_{1}(y), \\
u_{1}\left(x_{0}, y_{0}\right)=0, \\
u_{2}\left(x, x_{0}\right)=\beta_{2}(x), \quad u_{2}\left(y_{0}, y\right)=\tau_{2}(y), \\
u_{1}\left(x_{0}, y_{0}\right)=0,
\end{gathered}
$$

respectively where $\beta_{1}, \beta_{2}, \tau_{1}, \tau_{2} \in C_{\mathrm{rd}}\left(\mathbb{T}, \mathbb{R}^{n}\right)$,

$$
\left|\beta_{1}(x)+\tau_{1}(y)-\beta_{2}(x)-\tau_{2}(y)\right| \leq \mu,
$$

where $\mu \geq 0$ is a constant. Then

$$
\begin{aligned}
& \left|u_{1}(x, y)-u_{2}(x, y)\right|+\left|u_{1}^{\Delta_{2} \Delta_{1}}(x, y)-u_{2}^{\Delta_{2} \Delta_{1}}(x, y)\right| \\
& \quad \leq \frac{\mu}{1-d} e_{\bar{p}(x, y)}\left(x, x_{0}\right),
\end{aligned}
$$


for $(x, y) \in \Omega$, where

$$
\bar{p}(x, y)=\int_{y_{0}}^{y}\left[\frac{d}{1-d}+B(s, t)\right] \Delta t \Delta s,
$$

where $B(s, t)$ is defined by the right hand side of (18) replacing $b(x, y, m, n)$ and $p(x, y, m, n)$ by $(1 /(1-d)) p(x, y, m, n)$.

Proof. Let $w(x, y)=\left|u_{1}(x, y)-u_{2}(x, y)\right|+\mid u_{1}^{\Delta_{2} \Delta_{1}}(x, y)-$ $u_{2}^{\Delta_{2} \Delta_{1}}(x, y) \mid$ for $(x, y) \in \Omega$. We have

$$
\begin{aligned}
& w(x, y) \\
& \leq\left|\beta_{1}(x)+\tau_{1}(y)-\beta_{2}(x)-\tau_{2}(y)\right| \\
& +\int_{x_{0}}^{x} \int_{y_{0}}^{y} \mid f\left(s, t, u_{1}(s, t), u_{1}^{\Delta_{2} \Delta_{1}}(s, t), H\left(u_{1}\right)(s, t)\right) \\
& -f\left(s, t, u_{2}(s, t), u_{2}^{\Delta_{2} \Delta_{1}}(s, t), H\left(u_{2}\right)(s, t)\right) \mid \\
& \times \Delta t \Delta s
\end{aligned}
$$$$
+\mid f\left(x, y, u_{1}(x, y), u_{1}^{\Delta_{2} \Delta_{1}}(x, y), H\left(u_{1}\right)(x, y)\right)
$$$$
-f\left(x, y, u_{2}(x, y), u_{2}^{\Delta_{2} \Delta_{1}}(x, y), H\left(u_{2}\right)(x, y)\right) \mid
$$$$
+\mu+\int_{x_{0}}^{x} \int_{y_{0}}^{y}\left\{d \left[\left|u_{1}(s, t)-u_{2}(s, t)\right|\right.\right.
$$$$
\left.+\left|u_{1}^{\Delta_{2} \Delta_{1}}(s, t)-u_{2}^{\Delta_{2} \Delta_{1}}(s, t)\right|\right]
$$$$
+\int_{x_{0}}^{s} \int_{y_{0}}^{t} p(s, t, m, n)
$$$$
\times\left[\left|u_{1}(m, n)-u_{2}(m, n)\right|\right.
$$$$
\left.+\left|u_{1}^{\Delta_{2} \Delta_{1}}(m, n)-u_{2}^{\Delta_{2} \Delta_{1}}(m, n)\right|\right]
$$$$
\times \Delta n \Delta m\} \Delta t \Delta s
$$$$
+d\left[\left|u_{1}(x, y)-u_{2}(x, y)\right|\right.
$$$$
\left.+\left|u_{1}^{\Delta_{2} \Delta_{1}}(x, y)-u_{2}^{\Delta_{2} \Delta_{1}}(x, y)\right|\right]
$$$$
+\int_{x_{0}}^{x} \int_{y_{0}}^{y} p(x, y, m, n)
$$$$
\times\left[\left|u_{1}(m, n)-u_{2}(m, n)\right|\right.
$$$$
\left.+\left|u_{1}^{\Delta_{2} \Delta_{1}}(m, n)-u_{2}^{\Delta_{2} \Delta_{1}}(m, n)\right|\right] \Delta n \Delta m .
$$

From (47), we have

$$
\begin{aligned}
& w(x, y) \\
& \leq \frac{\mu}{1-d}+\frac{1}{1-d} \\
& \quad \times \int_{x_{0}}^{x} \int_{y_{0}}^{y}\{w(s, t)+p(x, y, s, t) w(s, t)
\end{aligned}
$$

$$
\left.+\int_{x_{0}}^{s} \int_{y_{0}}^{t} p(s, t, m, n) w(m, n) \Delta n \Delta m\right\}
$$

$\times \Delta t \Delta s$

Now, a suitable application of Lemma 2 to (48) yields the bound (45) which shows the dependency of solution of (1) on the initial boundary conditions.

Now, we consider initial boundary value problem (1) and (2) and the corresponding initial boundary value problem

$$
v^{\Delta_{2} \Delta_{1}}(x, y)=G\left(x, y, v(x, y), v^{\Delta_{2} \Delta_{1}}(x, y),(\bar{H} v(x, y))\right),
$$

with the initial boundary conditions

$$
v\left(x, x_{0}\right)=\bar{\beta}(x), \quad v\left(y_{0}, y\right)=\bar{\tau}(y), \quad v\left(x_{0}, y_{0}\right)=0,
$$

where

$$
\begin{aligned}
(\bar{H} v) & (x, y) \\
& =\int_{x_{0}}^{x} \int_{y_{0}}^{y} J\left(x, y, m, n, v(m, n), v^{\Delta_{2} \Delta_{1}}(m, n)\right) \Delta n \Delta m,
\end{aligned}
$$

$G \in C_{\mathrm{rd}}\left(\Omega \times \mathbb{R}^{n} \times \mathbb{R}^{n} \times \mathbb{R}^{n}, \mathbb{R}^{n}\right), J \in C_{\mathrm{rd}}\left(\Omega \times \Omega \times \mathbb{R}^{n} \times \mathbb{R}^{n}, \mathbb{R}^{n}\right)$, and $\bar{\beta}, \bar{\tau} \in C_{\mathrm{rd}}\left(\mathbb{T}, \mathbb{R}^{n}\right)$.

Now, we present the result which deals with continuous dependence of solution of initial boundary value problem (1) and (2) on the functions involved therein.

Theorem 8. Assume that the functions $f, g$ in (1) satisfy the conditions (30) and

$$
\begin{gathered}
|\beta(x)+\tau(y)-\bar{\beta}(x)-\bar{\tau}(y)| \\
+\int_{x_{0}}^{x} \int_{y_{0}}^{y} \mid f\left(s, t, v(s, t), v^{\Delta_{2} \Delta_{1}}(x, y),(H v)(s, t)\right) \\
\quad-G\left(s, t, v(s, t), v^{\Delta_{2} \Delta_{1}}(s, t),(\bar{H} v)(s, t)\right) \mid \\
\quad \times \Delta t \Delta s \\
+\mid f\left(x, v, v(x, y), v^{\Delta_{2} \Delta_{1}}(x, y),(H v)(x, y)\right) \\
-G\left(x, y, v(x, y), v^{\Delta_{2} \Delta_{1}}(x, y),(\bar{H} v)(x, y)\right) \mid \leq \epsilon
\end{gathered}
$$

where $f, g, \beta, \tau$ and $G, J, \bar{\beta}, \bar{\tau}$ are as in initial boundary value problem (1) and (2) and initial boundary value problem (49), (50), $\epsilon \geq 0$ is a constant, and $v(x, y)$ is a solution of initial boundary value problem (49) and (50). Then, solution (1) and (2) depends continuously on the functions involved therein. 
Proof. Let $w(x, y)=|u(x, y)-v(x, y)|+\mid u^{\Delta_{2} \Delta_{1}}(x, y)-$ $v^{\Delta_{2} \Delta_{1}}(x, y) \mid$ for $(x, y) \in \Omega$. We have

$$
\begin{aligned}
& w(x, y) \\
& \leq|\beta(x)+\tau(y)-\bar{\beta}(x)-\bar{\tau}(y)| \\
& \quad+\int_{x_{0}}^{x} \int_{y_{0}}^{y} \mid f\left(s, t, u(s, t), u^{\Delta_{2} \Delta_{1}}(s, t),(H u)(s, t)\right) \\
& \quad-f\left(s, t, v(s, t), v^{\Delta_{2} \Delta_{1}}(s, t),(H v)(s, t)\right) \mid
\end{aligned}
$$$$
\times \Delta t \Delta s
$$

$$
\begin{array}{r}
+\int_{x_{0}}^{x} \int_{y_{0}}^{y} \mid f\left(s, t, v(s, t), v^{\Delta_{2} \Delta_{1}}(x, y),(H v)(s, t)\right) \\
-G\left(s, t, v(s, t), v^{\Delta_{2} \Delta_{1}}(s, t),(\bar{H} v)(s, t)\right) \mid
\end{array}
$$$$
\times \Delta t \Delta s
$$

$$
\begin{array}{r}
+\mid f\left(x, y, u(x, y), u^{\Delta_{2} \Delta_{1}}(x, y),(H u)(x, y)\right) \\
-f\left(x, y, v(x, y), v^{\Delta_{2} \Delta_{1}}(x, y),(H v)(x, y)\right) \mid \\
+\mid f\left(x, y, v(x, y), v^{\Delta_{2} \Delta_{1}}(x, y),(H v)(x, y)\right) \\
-G\left(x, y, v(x, y), v^{\Delta_{2} \Delta_{1}}(s, t),(\bar{H} v)(s, t)\right) \mid
\end{array}
$$$$
\leq \epsilon+\int_{x_{0}}^{x} \int_{y_{0}}^{y}\{d[|u(s, t)-v(s, t)|
$$$$
\left.+\left|u^{\Delta_{2} \Delta_{1}}(s, t)-v^{\Delta_{2} \Delta_{1}}(s, t)\right|\right]
$$$$
+\int_{x_{0}}^{x} \int_{y_{0}}^{y} p(s, t, m, n)
$$$$
\times[|u(m, n)-v(m, n)|
$$$$
\left.+\left|u^{\Delta_{2} \Delta_{1}}(s, t)-v^{\Delta_{2} \Delta_{1}}(s, t)\right|\right]
$$$$
\times \Delta n \Delta m\} \Delta t \Delta s
$$$$
+d[|u(x, y)-v(x, y)|
$$$$
\left.+\left|u^{\Delta_{2} \Delta_{1}}(x, y)-v^{\Delta_{2} \Delta_{1}}(x, y)\right|\right]
$$$$
+\int_{x_{0}}^{x} \int_{y_{0}}^{y} p(x, y, m, n)
$$$$
\times[|u(m, n)-v(m, n)|
$$$$
\left.+\left|u^{\Delta_{2} \Delta_{1}}(m, n)-v^{\Delta_{2} \Delta_{1}}(m, n)\right|\right]
$$

$\times \Delta n \Delta m$
From (53), we have

$$
\begin{aligned}
& w(x, y) \\
& \leq \frac{\epsilon}{1-d} \\
& \quad+\frac{1}{1-d} \int_{x_{0}}^{x} \int_{y_{0}}^{y}\{w(s, t)+p(x, y, s, t) w(s, t) \\
& \left.\quad+\int_{x_{0}}^{s} \int_{y_{0}}^{t} p(s, t, m, n) w(m, n) \Delta n \Delta m\right\} \\
& \quad \times \Delta t \Delta s .
\end{aligned}
$$

Now, a suitable application of Lemma 2 yields

$$
w(x, y) \leq \frac{\epsilon}{1-d} e_{\bar{p}(x, y)}\left(x, x_{0}\right)
$$

for $(x, y) \in \Omega$, where $\bar{p}(x, y)$ is given by (43). From (35), it follows that solution of initial boundary value problem (1) and (2) depends continuously on the functions involved therein.

Remark 9. In this paper, we have studied the existence and uniqueness of solution of (1) using Banach fixed-point theorem. Here, we have offered simple and concise proofs of properties of solutions of (1). I believeed results given will serve as a model for further investigations.

\section{Acknowledgment}

The author is grateful to the anonymous referee whose comments and suggestions helped the author to improve the text.

\section{References}

[1] E. A. Bohner, M. Bohner, and F. Akin, "Pachpatte inequalities on time scales," Journal of Inequalities in Pure and Applied Mathematics, vol. 6, no. 1, article 6, 2005.

[2] M. Bohner and G. S. Guseinov, "Partial differentiation on time scales," Dynamic Systems and Applications, vol. 13, no. 3-4, pp. 351-379, 2004.

[3] M. Bohner and G. S. Guseinov, "Multiple integration on time scales," Dynamic Systems and Applications, vol. 14, no. 3-4, pp. 579-606, 2005.

[4] J. Hoffacker, "Basic partial dynamic equations on time scales," Journal of Difference Equations and Applications, vol. 8, no. 4, pp. 307-319, 2002.

[5] B. Jackson, "Partial dynamic equations on time scales," Journal of Computational and Applied Mathematics, vol. 186, no. 2, pp. 391-415, 2006.

[6] T. Kulik and C. C. Tisdell, "Volterra integral equations on time scales: basic qualitative and quantitative results with applications to initial value problems on unbounded domains," International Journal of Difference Equations, vol. 3, no. 1, pp. 103-133, 2008. 
[7] D. B. Pachpatte, "Explicit estimates on integral inequalities with time scale," Journal of Inequalities in Pure and Applied Mathematics, vol. 7, no. 4, article 143, 2005.

[8] D. B. Pachpatte, "Properties of solutions to nonlinear dynamic integral equations on time scales," Electronic Journal of Differential Equations, vol. 2008, no. 136, pp. 1-8, 2008.

[9] D. B. Pachpatte, "Integral inequalitys for partial dynamic equations on time scales," Electronic Journal of Differential Equations, vol. 2012, no. 50, pp. 1-17, 2012.

[10] M. H. Sarikaya, N. A. Yildirim, and K. I. Larslan, "Partial $\Delta$ differentiation for multivariable functions on $n$-dimensional time scales," Journal of Mathematical Inequalities, vol. 3, no. 2, pp. 277-291, 2009.

[11] C. C. Tisdell and A. Zaidi, "Basic qualitative and quantitative results for solutions to nonlinear, dynamic equations on time scales with an application to economic modelling," Nonlinear Analysis: Theory, Methods and Applications, vol. 68, no. 11, pp. 3504-3524, 2008.

[12] M. Bohner and A. Peterson, Dynamic Equations on Time Scales, Birkhäauser, Boston, Mass, USA, 2001.

[13] M. Bohner and A. Peterson, Advances in Dynamic Equations on Time Scales, Birkhäauser, Boston, Mass, USA, 2003.

[14] S. Hilger, "Analysis on measure chain-a unified approch to continuous and discrete calculus," Results in Mathematics, vol. 18, no. 1-2, pp. 18-56, 1990.

[15] R. A. C. Ferreira and D. F. M. Torres, "Some linear and nonlinear integral inequalities on time scales in two independent variables," Nonlinear Dynamics and Systems Theory, vol. 9, no. 2, pp. 161-169, 2009. 


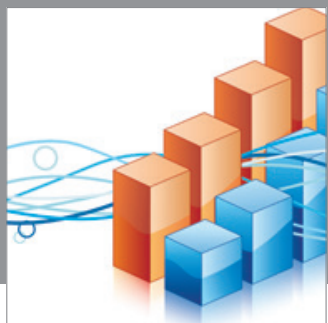

Advances in

Operations Research

mansans

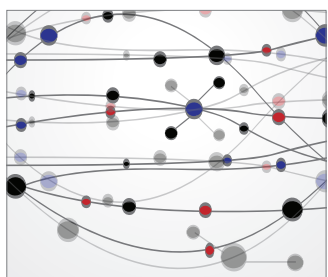

The Scientific World Journal
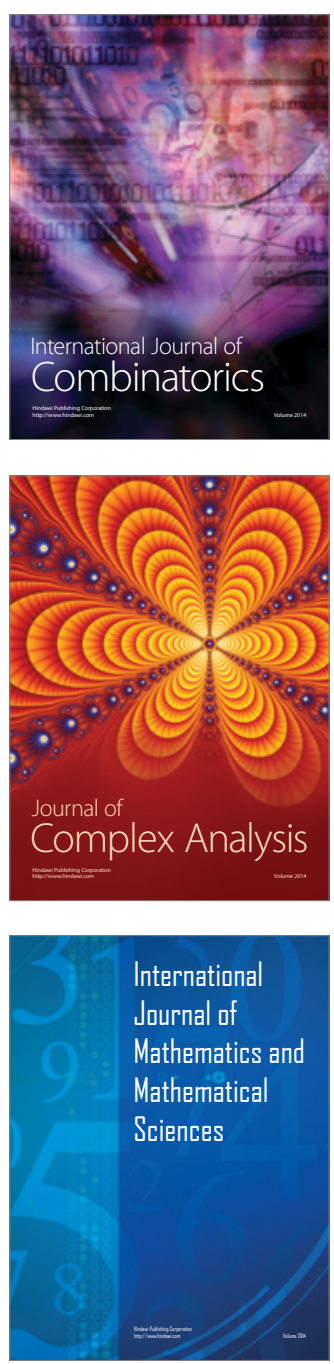
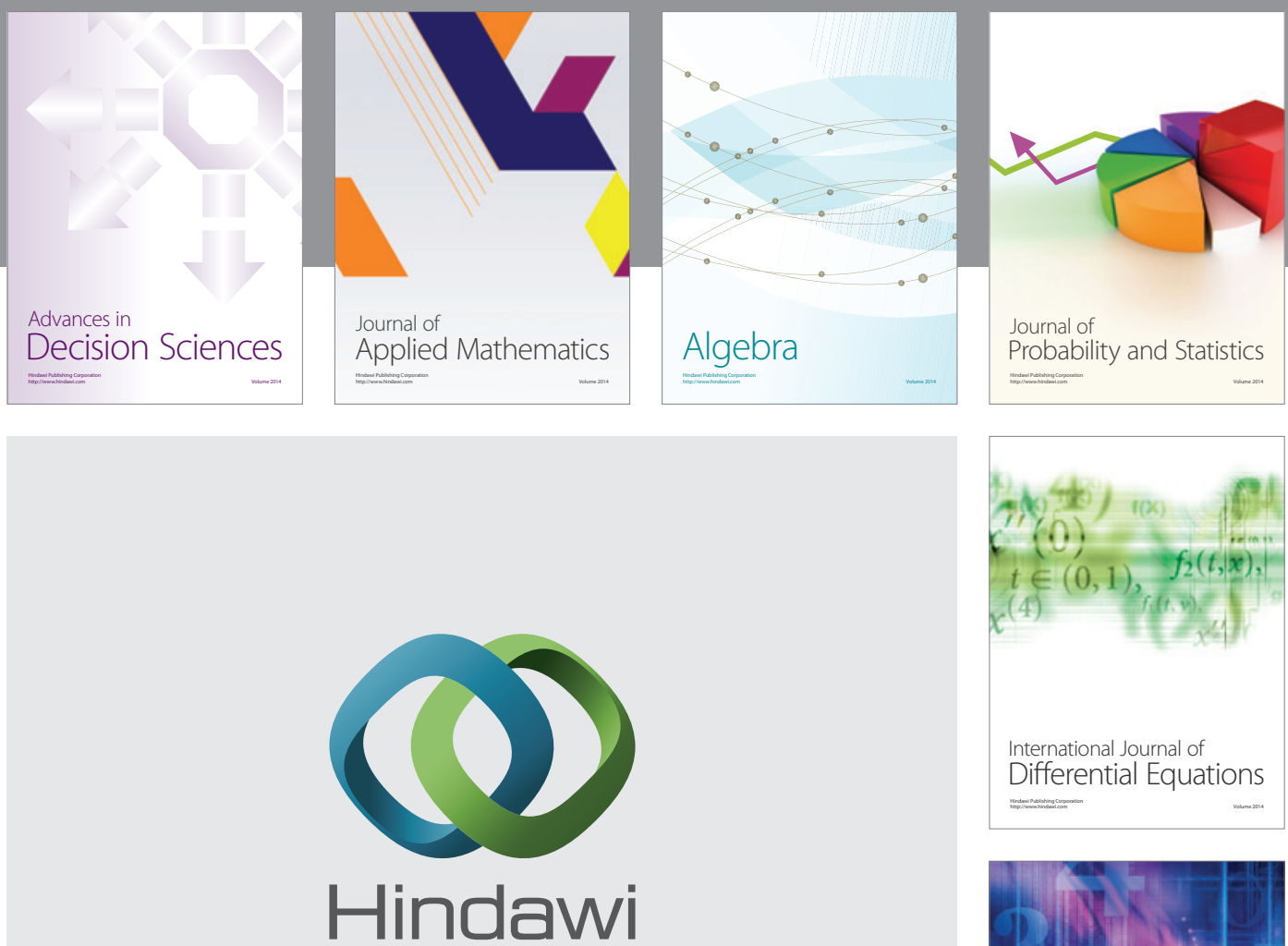

Submit your manuscripts at http://www.hindawi.com
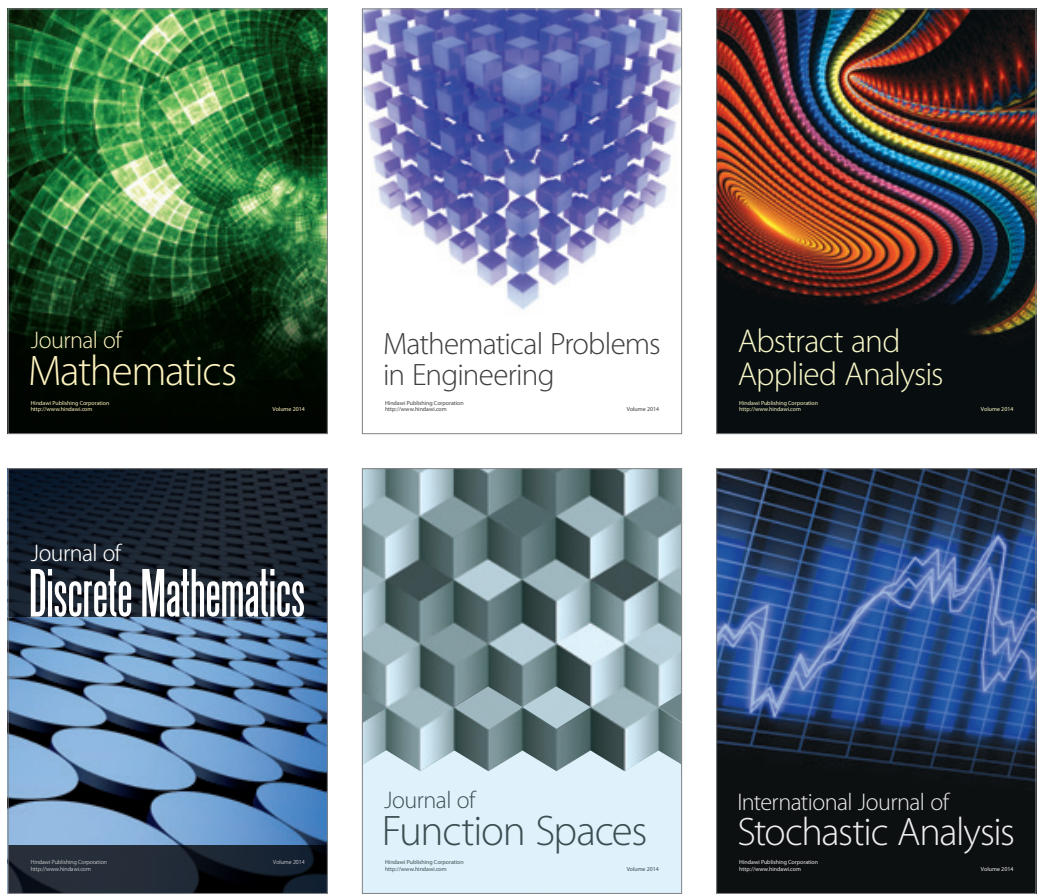

Journal of

Function Spaces

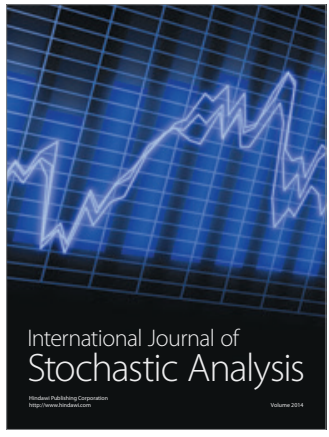

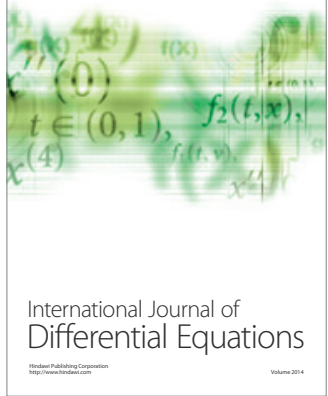
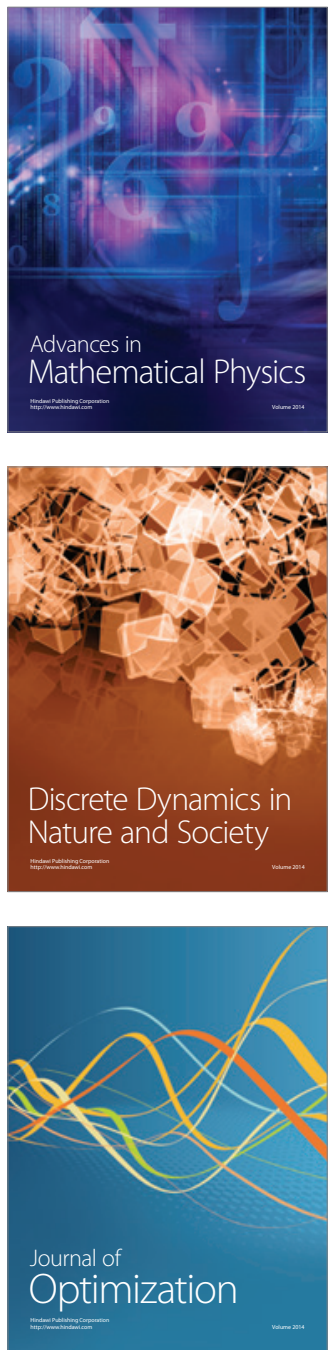